\title{
SU(2) Lattice Gluon Propagator and Potential Models
}

\author{
Willian Matioli Serenone ${ }^{* \dagger}$ \\ IFSC, University of São Paulo \\ CP 369 CEP 13560-970, São Carlos SP, Brazil \\ E-mail: willian.matioli@gmail.com

\section{Attilio Cucchieri} \\ IFSC, University of São Paulo \\ CP 369 CEP 13560-970, São Carlos SP, Brazil \\ E-mail: attiliodifsc.usp.br

\section{Tereza Mendes} \\ IFSC, University of São Paulo \\ CP 369 CEP 13560-970, São Carlos SP, Brazil \\ E-mail: mendeseifsc.usp.br
}

We study the bottomonium spectrum using a potential model. Our potential incorporates lattice results for the gluon propagator, obtained from simulations of pure SU(2) gauge theory in Landau gauge. The mass of the bottom quark is left as a free parameter. The resulting spectrum is compared to the case of the Coulomb plus Linear (or Cornell) Potential.

31st International Symposium on Lattice Field Theory - LATTICE 2013

July 29 - August 3, 2013

Mainz, Germany

\footnotetext{
* Speaker.

${ }^{\dagger}$ We acknowledge FAPESP for financial support, grant number 2012/04811-0.
} 


\section{Introduction}

The goal of the present study is to obtain the spectrum of bottomonium states using a potentialmodel approach, including nonperturbative input from lattice simulations. The bottomonium spectrum is a rich one, whose spacings between fundamental levels are similar to those of the charmonium. This motivates the description by a common potential model [1]. Also, the mass of the bottom quark is large in comparison with the system energy (the same happens, to a lesser degree, with charmonium). This allows us to make a nonrelativistic approximation to the BetheSalpeter equation used in the description of bound states of two fermions (see e.g. Ref. [2] and [3]). More precisely, one makes an instantaneous approximation to the wave function (which leads to the Salpeter equation), a nonrelativistic approximation to the kinetic term in the Hamiltonian and a local approximation to the potential. After these approximations, the equation reduces to the Schrödinger equation.

We require the potential to respect some physical characteristics of the quark-antiquark interactions in QCD. This is usually accomplished by a combination of two behaviors. The first one refers to the quark-antiquark interaction in the one-gluon-exchange (OGE) approximation (related to quark-antiquark scattering inside the meson) and is of perturbative nature. The second one, the property of confinement, may be modeled by a linearly rising function, inspired by lattice QCD simulations (see Refs. [4], [5] and [6, Fig. 5]). If the free vector-boson propagator is used, the obtained potential will be the "Coulomb plus linear" or Cornell potential

$$
V(r)=-\frac{4}{3} \frac{\alpha_{s}}{r}+F_{0} r
$$

Other commonly used potentials are listed in [7, Section 7]. Here we substitute the free gluon propagator with the one provided in Ref. [8], obtained from lattice simulations of pure SU(2) gauge theory in Landau gauge. This introduces nonperturbative features into the scattering term of the potential.

In Section 2 we briefly describe the procedure for calculating the potential from the gluon propagator. In Section 3 we detail our method for solving the Schrödinger equation for an arbitrary potential and therefore obtaining the bottomonium spectrum. The results of this method are presented in Section 4 and we present our conclusions in Section 5. Preliminary results of our study were reported in [9].

\section{Brief Review of Potential Models}

The use of potential models in the study of heavy quarkonia is based on the assumption that the interaction between a heavy quark (namely the charm or the bottom quark) and its antiquark may be described by a potential. This is inspired by the fact that the Coulomb potential, which may be obtained as a limiting case from QED, explains with great accuracy bound states of nonrelativistic systems such as atoms or the positronium.

To obtain the Coulomb potential, one starts by considering an elastic $e^{-} e^{+}$scattering process. Applying perturbation theory in the first-order Born approximation, we obtain the scattering-matrix element $S_{f i}$

$$
S_{f i} \equiv\langle f \mid i\rangle=\delta_{f i}+i(2 \pi)^{4} \delta^{(4)}(Q-P) T_{f i},
$$


where $Q$ and $P$ correspond respectively to the final and initial total momentum and $T_{f i}$ is the scattering amplitude, which can be computed through Feynman rules. There are two Feynman diagrams contributing to it, which are shown in Fig. 1.
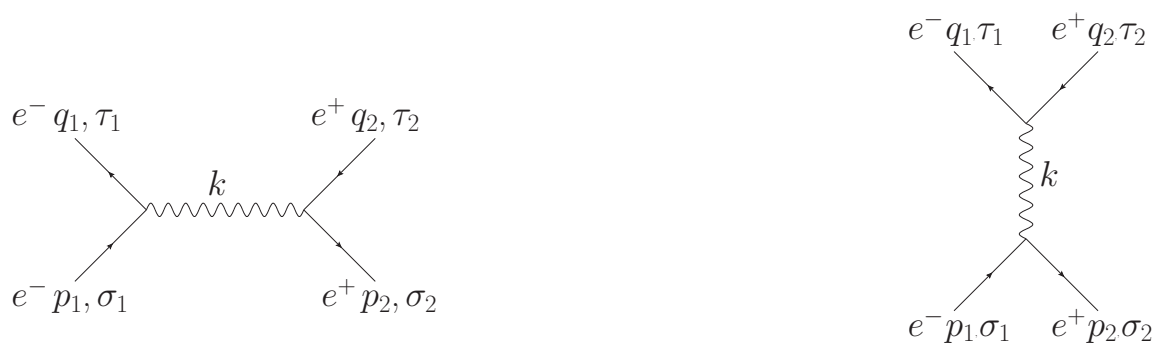

Figure 1: Feynman diagrams leading to the Coulomb potential in QED.

These diagrams result in the following scattering amplitude

$$
\begin{aligned}
T_{f i}=\frac{1}{(2 \pi)^{6}} \frac{m^{2}}{\sqrt{E_{p_{1}} E_{p_{2}} E_{q_{1}} E_{q_{2}}}}[ & -e^{2} \bar{u}\left(q_{1}, \tau_{1}\right) \gamma^{\mu} u\left(p_{1}, \sigma_{1}\right) P_{\mu v}(k) \bar{v}\left(p_{2}, \sigma_{2}\right) \gamma^{v} v\left(q_{2}, \tau_{2}\right) \\
& \left.+e^{2} \bar{v}\left(p_{2}, \sigma_{2}\right) \gamma^{\mu} u\left(p_{1}, \sigma_{1}\right) P_{\mu v}(k) \bar{u}\left(q_{1}, \tau_{1}\right) \gamma^{v} v\left(q_{2}, \tau_{2}\right)\right],
\end{aligned}
$$

where we follow the notation in [7].

We then make the nonrelativistic approximation, i.e. we impose the kinetic energy of the system to be much smaller than its rest energy $(p \ll m \cong E)$. If the usual photon propagator $P_{\mu \nu}(k)=g_{\mu \nu} / k^{2}$ is used, we see that the contribution of the second term on the r.h.s. of Eq. (2.2), which comes from the annihilation diagram, is negligible compared to the first term. Thus, performing a spatial Fourier transform, we recover the familiar Coulomb potential

$$
V(\mathbf{r})=-(2 \pi)^{3} \int \exp (-i \mathbf{k} \cdot \mathbf{r}) T_{f i}(k) d^{3} r=-\frac{1}{(2 \pi)^{3}} \int \exp (-i \mathbf{k} \cdot \mathbf{r}) \frac{e^{2}}{\mathbf{k}^{2}} d^{3} r=-\frac{e^{2}}{r} .
$$

This potential may be used to model the interaction in the positronium. Since we know that the binding energies will be of the order of $\mathrm{eV}$, while the electron and positron masses are approximately $0.5 \mathrm{MeV}$, we can expect that the nonrelativistic approximations for the potential will hold. We may use this potential in the Schrödinger equation to obtain the energy spectrum of the system. Notice that the nonrelativistic approximation completely removes spin dependencies of the potential.

In our work we follow the same procedure, replacing the electron-positron pair by a quarkantiquark pair and the photon by a gluon. For the gluon propagator we use an expression obtained from fits of lattice data for pure SU(2) gauge theory in Landau gauge, given in Ref. [8]. The limitation of this method is that, since we apply perturbation theory to obtain the potential, $V(r)$ is not expected to be a confining potential, even though the propagator used is obtained nonperturbatively. We model confinement, as usual, by adding to the potential a linearly rising term $F_{0} r$.

\section{Method for Obtaining Bottomonium Masses}

We expect that a typical choice of propagator will result in a central potential if we use the approximations in Section 2. Since our system contains only two particles, the Hamiltonian will 
be essentially the same as the one of the hydrogen atom, written in terms of relative coordinates. We use separation of variables to isolate the angular dependence of the wave function (which will be given by the spherical harmonics) and the variable substitution $R(r)=f(r) / r$ to obtain the Ordinary Differential Equation (ODE) for $f(r)$

$$
\frac{d^{2} f}{d r^{2}}+2 \mu\left[E-V(r)-2 m_{b}-\frac{l(l+1)}{2 \mu r^{2}}\right] f(r)=0,
$$

where $\mu$ is the reduced mass and $m_{b}$ is the mass of the bottom quark. Notice the addition of the rest mass of the particles, which will allow us to compare the eigenenergy directly with masses in Ref. [10].

Since the potential is arbitrary, it will not usually be possible to find an analytic expression for the eigenenergies. We therefore use a numerical approach. The algorithm consists in the following steps:

1. Finding a likely range for the eigenenergies and discretizing this interval in $N$ steps separated by $d E$. (We fix the range using the experimental values for the lowest and the highest energy states.)

2. Numerically solving the ODE in Eq. (3.1) to obtain the function $f(r)$ for each energy. We use the Numerov method (see Ref. [11, Chapter 3]).

3. Estimating the eigenenergy using the boundary conditions. The functions $f(r)$ will generally diverge to $\pm \infty$, since our guess for $E$ in Eq. (3.1) is not an eigenenergy. If we find that the sign of this divergence is reversed when changing from $E_{n, i}$ to $E_{n+1, i}$, the $i$ th eigenenergy will be estimated by $\left(E_{n, i}+E_{n+1, i}\right) / 2$. The error is taken as $d E / 2$.

Note that the only free parameter in the potential is the string force $F_{0}$, but we also leave free the mass of the bottom quark since, at present, it is not well determined. In fact, different approaches give different results for $m_{b}$ (for instance, Ref. [10] has two values for it). To find the best values for these parameters, we adopt a similar strategy used in the calculation of the eigenenergies described above: we set a range where it is believed the values of the parameters may be and discretize it. We then compute the eigenenergies for each proposed set of parameters and select the one that best describes the observed spectrum. The criteria for choosing a set of parameters with this property is to look for the set that minimizes the residual

$$
R(\text { Parameters })=\sum_{i}\left(E_{i}-E_{i, \text { Experimental }}\right)^{2}
$$

\section{Results}

Following the discussion in Section 2, the Feynman diagrams for the bottomonium will be similar to the ones in the $e^{-} e^{+}$scattering shown in Fig. 1. This similarity means that the scattering matrix will have the same structure. The main difference will be extra factors due to the $S U(3)$ symmetry of QCD. Considering that the gluon propagator is color-diagonal, i.e. $P_{\mu \nu}^{a b}(k) \propto \delta^{a b}$, the 
color factors contributing to the scattering diagram and the annihilation diagram will be respectively

$$
\begin{aligned}
c_{1, f}^{\dagger} \frac{\lambda^{a}}{2} c_{1, i} c_{2, i}^{\dagger} \frac{\lambda^{a}}{2} c_{2, f} & =\frac{4}{3} \\
c_{2, i}^{\dagger} \frac{\lambda^{a}}{2} c_{1, i} c_{1, f}^{\dagger} \frac{\lambda^{a}}{2} c_{2, f} & =0,
\end{aligned}
$$

where the vectors $c_{1,2}$ represent the color states and $\lambda^{a}$ are the Gell-Mann matrices. Notice that here the contribution from the annihilation diagram is exactly zero.

The propagator from Ref. [8] has the form

$$
P_{\mu v}^{a b}(k)=\frac{C\left(s^{2}+k^{2}\right)}{t^{2}+u^{2} k^{2}+k^{4}}\left(\delta_{\mu v}-\frac{k_{\mu} k_{v}}{k^{2}}\right) \delta^{a b} .
$$

(Values for the normalization constant $C$ and the parameters $s, t, u$ are given in [8].)

By analogy with the QED case considered before, we keep only the first term of the above tensor structure. Also, this propagator is obtained for Euclidean time and therefore we need to make the transformation $\delta_{\mu v} \rightarrow g_{\mu v}$. (Without this transformation the perturbative term would be repulsive.) Furthermore, we approximate all energies to the particle mass, which implies $m^{2} / \sqrt{E_{p_{1}} E_{p_{2}} E_{q_{1}} E_{q_{2}}}=1$. With these approximations, we have

$$
T_{f i}(k)=-\frac{1}{(2 \pi)^{6}}\left[\frac{4}{3} g_{s}^{2} \bar{u}\left(q_{1}, \tau_{1}\right) \gamma^{\mu} u\left(p_{1}, \sigma_{1}\right) g_{\mu v} \frac{C\left(s^{2}+k^{2}\right)}{t^{2}+u^{2} k^{2}+k^{4}} \bar{v}\left(p_{2}, \sigma_{2}\right) \gamma^{v} v\left(q_{2}, \tau_{2}\right)\right] .
$$

We need now to compute the factors coming from the spinors. When we impose that the particles be nonrelativistic, we obtain

$$
\begin{aligned}
\bar{u}\left(q_{1}, \tau_{1}\right) \gamma^{\mu} u\left(p_{1}, \sigma_{1}\right) & =\delta^{\mu 0} \delta_{\sigma_{1} \tau_{1}} \\
\bar{v}\left(p_{2}, \sigma_{2}\right) \gamma^{v} v\left(q_{2}, \tau_{2}\right) & =\delta^{v 0} \delta_{\sigma_{2} \tau_{2}} .
\end{aligned}
$$

The final scattering amplitude is

$$
T_{f i}(k)=\frac{1}{(2 \pi)^{6}}\left[\frac{4}{3} g_{s}^{2} \frac{C\left(s^{2}+k^{2}\right)}{t^{2}+u^{2} k^{2}+k^{4}}\right] .
$$

We proceed to computing the Fourier transform. The angular integral is easily solved by assuming that the point $\mathbf{r}$ lies on the z-axis. The radial integration can be easily computed through the residue method. Notice that Eq. (4.7) has four simple poles, one in each quadrant of the complex plane. The angular integration does not add any new pole to it. These poles are distributed in such a way that, once one of them is obtained, it is possible to reproduce all others by complex conjugation and/or multiplication by -1 . These symmetries allow one to express the four terms coming from the residue calculation around each pole as a single term, dependent only on the pole of the first quadrant. The potential becomes

$$
\begin{aligned}
V(r) & =-\frac{4}{3} \frac{g_{s}^{2}}{\pi^{2} r} \Re\left[\frac{C\left(s+k_{1}^{2}\right) \exp \left(i k_{1} r\right)}{4 k_{1}^{2}-u^{4}}\right], \\
k_{1} & =i \sqrt{t} \exp \left[-\frac{i}{2} \arctan \left(\frac{\sqrt{4 t^{2}-u^{4}}}{u^{4}}\right)\right] .
\end{aligned}
$$


We implement the algorithm described in Section 3 for quark masses from $4.1 \mathrm{GeV}$ through 4.8 GeV (which includes both masses listed in Ref. [10]). For the string-tension parameter $F_{0}$, we search from $0.1 \mathrm{GeV}^{2}$ through $0.3 \mathrm{GeV}^{2}$. We apply the same algorithm using the Coulomb plus linear potential for comparison. The results can be found in Table 1.

\begin{tabular}{||c|c||c|c||c|c||}
\hline \multicolumn{2}{||c||}{$\begin{array}{c}m_{b}(\overline{\mathrm{MS}})=4.18(3) \mathrm{GeV} \\
m_{b}(1 \mathrm{~S})=4.66(3) \mathrm{GeV} \\
\text { See }[10]\end{array}$} & $\begin{array}{c}\text { Potential from } \\
\text { Lattice Propagator }\end{array}$ & $\begin{array}{c}F_{0}=0.2118(1) \mathrm{GeV}^{2} \\
m_{b}=4.5977(1) \mathrm{GeV} \\
R=0.0436\end{array}$ & $\begin{array}{c}\text { Coulomb plus } \\
\text { Linear Potential }\end{array}$ & $\begin{array}{c}F_{0}=0.2136(1) \mathrm{GeV}^{2} \\
m_{b}=4.6090(1) \mathrm{GeV}^{2} \\
R=0.0475\end{array}$ \\
\hline $\begin{array}{c}\text { Particle } \\
\text { State }\end{array}$ & $\begin{array}{c}\text { Experimental } \\
\text { Mass(GeV) }\end{array}$ & $\begin{array}{c}\text { Calculated Mass } \\
\left( \pm \mathbf{3} \times \mathbf{1 0}^{-\mathbf{4}} \mathbf{G e V}\right)\end{array}$ & $\begin{array}{c}\text { Deviation from } \\
\text { Experiment }(\mathbf{G e V})\end{array}$ & $\begin{array}{c}\text { Calculated Mass } \\
\left( \pm \mathbf{3} \times \mathbf{1 0}^{-\mathbf{4}} \mathbf{G e V}\right)\end{array}$ & $\begin{array}{c}\text { Deviation from } \\
\text { Experiment }(\mathbf{G e V})\end{array}$ \\
\hline 1S* & $9.42565(153)$ & 9.5763 & 0.1507 & 9.5793 & 0.1528 \\
\hline 2S & $10.02326(31)$ & 10.0071 & 0.0162 & 10.0029 & 0.0204 \\
\hline 3S & $10.3552(5)$ & 10.3317 & 0.0235 & 10.3293 & 0.0259 \\
\hline 4S & $10.5794(12)$ & 10.6107 & 0.0313 & 10.6119 & 0.0325 \\
\hline 5S & $10.876(11)$ & 10.8633 & 0.0127 & 10.8675 & 0.0085 \\
\hline 6S & $11.019(8)$ & 11.0973 & 0.0783 & 11.1045 & 0.0855 \\
\hline 1P* & $9.89076(82)$ & 9.8595 & 0.0313 & 9.8565 & 0.0343 \\
\hline 2P* & $10.25410(94)$ & 10.2033 & 0.0508 & 10.2009 & 0.0532 \\
\hline 3P & $10.530(14)$ & 10.4943 & 0.0357 & 10.4949 & 0.0351 \\
\hline 1D & $10.1637(14)$ & 10.0743 & 0.0894 & 10.0683 & 0.0954 \\
\hline
\end{tabular}

Table 1: Comparison between the results obtained for the potential extracted using the lattice gluon propagator and the usual Coulomb plus Linear potential. Notice that the states marked with "** are actually an average of states with different spin but same orbital angular momentum.

We remark that our obtained values for the quark mass agree much better with the $m_{b}(1 \mathrm{~S})$ value from Ref. [10]. The small difference between the results for our potential and for the Coulomb plus linear potential can traced to the fact that the two potentials are nearly identical, as show in Fig. 2.

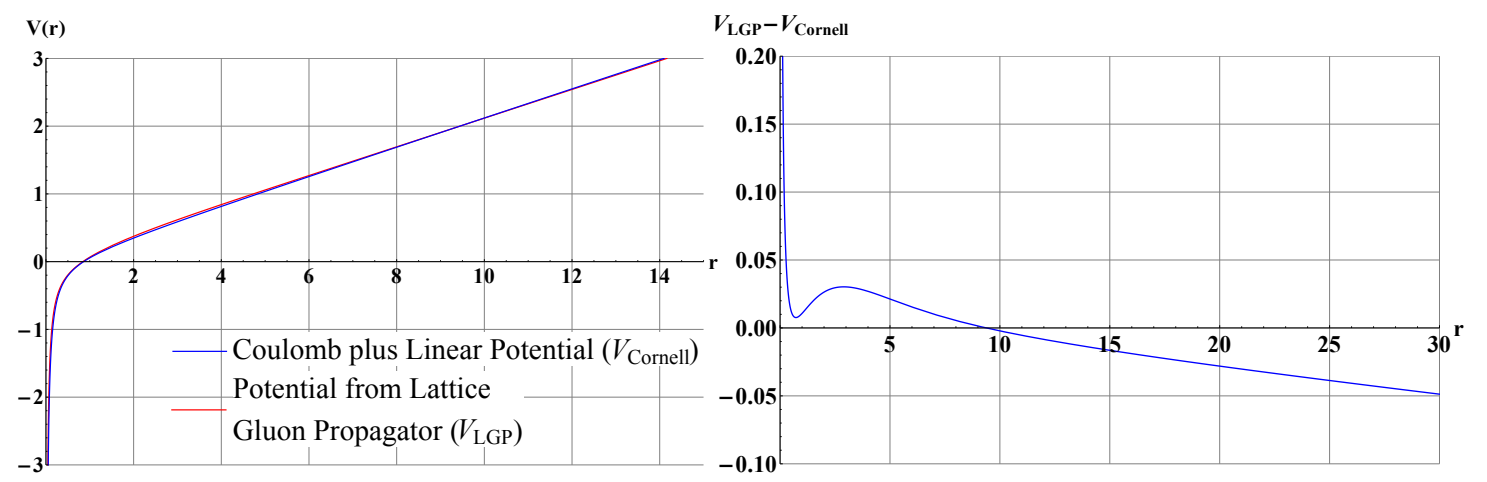

Figure 2: Plot of the potential obtained using the lattice gluon propagator and comparison with the Cornell potential (left panel). The computed string tension is only slightly different for the two cases. We also plot the difference between these two potentials (right panel). In both cases the potentials are given in $\mathrm{GeV}$ and the distances $r$ in $\mathrm{GeV}^{-1}$. 


\section{Conclusion}

We compute the bottomonium spectrum using a potential obtained from lattice simulations of the gluon propagator and compare to results using the Coulomb plus linear potencial. We find very similar behavior, with slightly better agreement with experimental data in the former case. This might indicate that, although the perturbative treatment removes most of the nonperturbative features of the scattering contribution to the potential, a small part of them survives this treatment.

We note that the propagator we used was computed for pure $\mathrm{SU}(2)$ gauge theory, instead of (pure) QCD. (Note also that this propagator is determined up to a global constant $C$, fixed by normalization.) The good agreement of our results with the experimental spectrum suggests that it is enough to take into account the SU(3) color structure by including the usual color factor in the calculation (see Eq. (4.1)).

In our study we do not include effects of spin-spin interaction or spin-orbit interactions, which can lead to splitting of some energy levels. We have as well additional errors due to our nonrelativistic approach. The main limitation, however, is clearly the use of the OGE approximation in the scattering calculation, and the need to include the confining term by hand. We nevertheless believe that the method is useful, especially if an application beyond the OGE approximation can be made. (We note that a different point of view is presented in a similar study reported in Ref. [12].)

\section{References}

[1] C. Patrignani, T. K. Pedlar, and J. L. Rosner, Recent Results in Bottomonium, arXiv: 1212.6552.

[2] J. Bijtebier and J. Broekaert, On the three-dimensional reductions of the Bethe-Salpeter equation and their one-body limits (two fermion case), J.Phys. G22 (1996) 559-578.

[3] A. Bernardini and C. Dobrigkeit, The Charmonium and bottomonium mass spectroscopy with a simple approximation of the kinetic term, J.Phys. G29 (2003) 1439-1449, [hep-ph/ 0611336 ].

[4] A. De Rujula, H. Georgi, and S. Glashow, Hadron Masses in a Gauge Theory, Phys.Rev. D12 (1975) $147-162$.

[5] SESAM Collaboration Collaboration, G. S. Bali, H. Neff, T. Duessel, T. Lippert, and K. Schilling, Observation of string breaking in QCD, Phys.Rev. D71 (2005) 114513, [hep-lat/ 0505012 ].

[6] M. Donnellan, F. Knechtli, B. Leder, and R. Sommer, Determination of the Static Potential with Dynamical Fermions, Nucl.Phys. B849 (2011) 45-63, [arXiv: 1012 .3037].

[7] W. Lucha, F. Schoberl, and D. Gromes, Bound states of quarks, Phys.Rept. 200 (1991) 127-240.

[8] A. Cucchieri, D. Dudal, T. Mendes, and N. Vandersickel, Modeling the Gluon Propagator in Landau Gauge: Lattice Estimates of Pole Masses and Dimension-Two Condensates, Phys.Rev. D85 (2012) 094513, [arXiv:1111.2327].

[9] W. M. Serenone and T. Mendes, Potential model for the bottomonium, AIP Conf.Proc. 1520 (2013) 364-366.

[10] Particle Data Group Collaboration, J. Beringer et al., Review of Particle Physics (RPP), Phys.Rev. D86 (2012) 010001.

[11] S. Koonin and D. Meredith, Computational Physics: Fortran Version. Perseus Books Group, 1998.

[12] P. Gonzalez, V. Mathieu, and V. Vento, Heavy meson interquark potential, Phys.Rev. D84 (2011) 114008, [arXiv:1108.2347]. 